\title{
Dentate gyrus is necessary for disambiguating similar object-place representations
}

\author{
Inah Lee $\mathrm{e}^{1,3}$ and Frances Solivan ${ }^{2}$ \\ ${ }^{1}$ Department of Brain and Cognitive Sciences, Seoul National University, Seoul 151-742, Korea; ${ }^{2}$ Department of Psychology, University \\ of lowa, lowa City, lowa 52242, USA
}

\begin{abstract}
Objects are often remembered with their locations, which is an important aspect of event memory. Despite the well-known involvement of the hippocampus in event memory, detailed intrahippocampal mechanisms are poorly understood. In particular, no experimental evidence has been provided in support of the role of the dentate gyrus (DG) in disambiguating such events, even though computational models suggest otherwise. In the current study, rats encountered multiple objects in different locations and were required to discriminate the object-place paired associates for reward. Specifically, two different objects appeared in one of two locations (arms in a radial maze) that were relatively close to each other. Different objects were rewarded depending on the arm in which the objects appeared. The rats with colchicine-based, dorsal DG (dDG) lesions showed severe and sustained impairment in disambiguating the objects compared with controls (Experiment 1). The dDG-lesioned rats were normal, however, in discriminating four different objects presented (Experiment 2) in the same locations as in Experiment 1. Finally, when the two different objects used in Experiment 1 were presented at two remote locations (Experiment 3) involving less overlap between arm-associated contextual cues, the dDG-lesioned animals showed initial deficits in discriminating the objects, but gradually relearned the task, in contrast to the sustained deficits observed in Experiment 1 . These results collectively suggest that the DG is necessary when the similarity is maximal between object-place paired associates due to overlapping object and/or spatial information, whereas its role becomes minimal as the overlap in either object or spatial information decreases.
\end{abstract}

The hippocampus and its associated regions are important in remembering spatial contexts and their associated events as discrete memories (O'Keefe and Nadel 1978; Kim and Fanselow 1992). One of the computational problems involved in this process is to disambiguate altered neural representations from the original memory representations formed at the time of encoding the events (Marr 1971; McNaughton and Morris 1987; O'Reilly and McClelland 1994). It is not uncommon that one needs to retrieve a specific memory representation of a particular person seen at a specific place, separately from many paired-associate representations previously formed between that person and other locations. It is largely unknown how the hippocampal networks form specific memory representations for multiple events that share common components and disambiguate those representations from each other when retrieving a specific target memory representation.

Among hippocampal subfields, computational models have highlighted the essential role of the dentate gyrus (DG) for disambiguating similar events. This so-called pattern separation refers to the computational process for making representations for similar input patterns more orthogonal to each other for better discrimination (Marr 1971; McNaughton and Morris 1987; O'Reilly and McClelland 1994). O'Reilly and McClelland (1994) have emphasized that feeding information through the multilayer structures from the entorhinal cortex to CA3 via DG facilitates pattern separation. Treves and Rolls (1992) have also argued that the key to forming a new pattern of firing in an autoassociative network such as CA3 for each event is in the connections between granule cells in DG and pyramidal cells in CA3 via the mossy fibers.

\footnotetext{
${ }^{3}$ Corresponding author.
}

E-mail inahlee@snu.ac.kr; fax 82-2-8719129.

Article is online at http://www.learnmem.org/cgi/doi/10.1101/Im.1678210.
Experimental efforts have been made to test these computational hypotheses regarding the DG function (Gilbert et al. 2001; Gilbert and Kesner 2003; Lee and Kesner 2004a,b; Leutgeb et al. 2007; McHugh et al. 2007). However, the involvement of DG in dissociating similar event memories composed of object-place paired associates has never been shown in the literature. Since associating objects and their locations has been considered an important task for studying the role of the hippocampus in episodic memory (Gaffan and Parker 1996; Vargha-Khadem et al. 1997; Bachevalier and Nemanic 2008), we tested whether the previously reported role of DG in orthogonalizing spatial representations also plays a vital role in discriminating similar object-place paired associates (Fig. 1).

\section{Results}

\section{Colchicine-based, dorsal DG lesions}

As previously described (Mundy and Tilson 1990; Xavier et al. 1999; Lee and Kesner 2004a,b; Jerman et al. 2005), colchicine injected into the dorsal DG (dDG; bregma -2.0 through $-4.0 \mathrm{~mm}$ ) produced severe and fairly selective damage in $\mathrm{dDG}$ and hilar regions but spared most pyramidal neurons histologically identified in CA1 and CA3 in the DG-lesion group (Fig. 2). No damage in the hippocampus was observed in the control group. The relative volumetric damage in dDG in the DG-lesion group was estimated to be $\sim 95 \%$ of the control group. There was $\sim 25 \%$ of damage in the principal cell layers of CA regions in the dorsal hippocampus in the DG-lesion group compared with controls. In both lesion groups, minor damages in the overlying cortices were observed because of the penetrations made for injection needles. These results, overall, match previous findings with intrahippocampal injections of colchicine (Xavier et al. 1999; Lee and Kesner 2004a,b). 


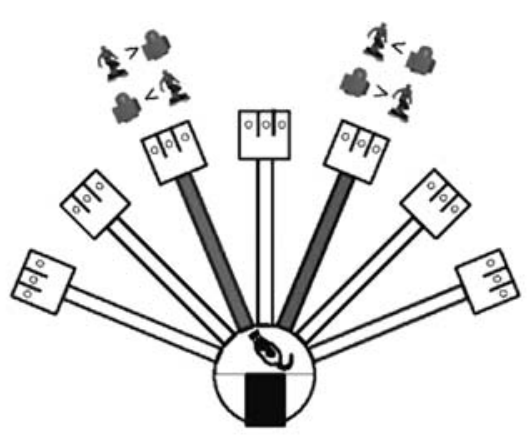

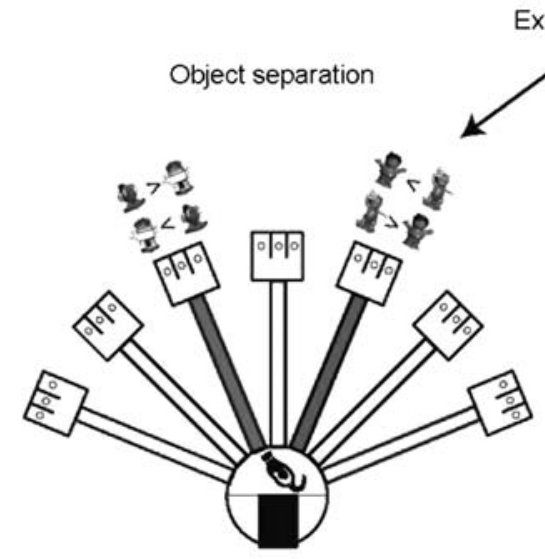

Experiment 2

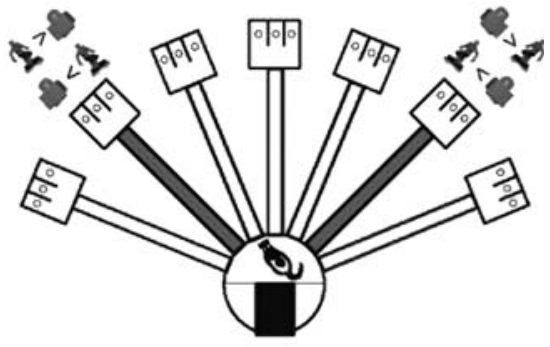

Experiment 3

Figure 1. Behavioral paradigm. Experiment 1: overview of the radial arm maze. Arms 3 and 5 (shown in gray for illustrative purposes only) were used, and the same pair of different objects was presented in either arm per trial. Object locations in each arm were counterbalanced as shown. Spider-Man was always rewarded (indicated by ">") in arm 3 and the LEGO block was rewarded in arm 5. Experiment 2: same as Experiment 1, except that the objects presented in arms 3 and 5 were different. Experiment 3: same as Experiment 1, except for the arms in which the two objects were presented; arms 2 and 6 were used.

\section{Severe impairment of object discrimination with dDG lesions in the biconditional task}

In Experiment 1, the rats were tested after surgery in the same biconditional object-place paired-associate task previously used for the pre-surgical training. Detailed descriptions of the task can be found in the Materials and Methods. Briefly, in this task, a pair of two different objects (Spider-Man and LEGO block, Fig. 1) was presented in two of the arms of a maze and a certain object of the pair was rewarded only in a particular arm (Spider-Man in arm 3 and LEGO block in arm 5, Fig. 1). Thus, the animals were required to process both object and space conditions at the same time (i.e., biconditional task) to make a correct response.

The control group maintained the pre-surgical performance level after surgery and further increased their performance to almost 95\% (Fig. 3A). The DG-lesion group, however, performed nearly at chance level throughout the entire testing period after surgery. An ANOVA with repeated measures showed significant effects of the lesion group $\left(F_{(1,10)}=445.4, P<0.0001\right)$, postsurgical training day $\left(F_{(5,50)}=8.5, P<0.0001\right)$, and the interaction between the two variables $\left(F_{(5,50)}=3.0, P<0.05\right)$. To eliminate the possibility that dDG lesions might have affected the capability of perceiving and discriminating objects at sensory-motor levels, we further tested four rats in each lesion group in a simple objectdiscrimination task. In this task, only a fixed, new arm (arm 4) was used and a pair of different new objects was presented in each trial (with food wells occupied by the two objects counterbalanced across trials). The number of days required to reach criterion performance $(75 \%)$ were not significantly different between the two lesion groups (data not shown; $t_{(6)}=$ 1.4, $P>0.1)$, confirming that the animals with colchicine-based dDG lesions were able to discriminate threedimensional objects when the object discrimination did not require conditional judgments on the basis of the spatial locations associated with the objects.

\section{Intact object discrimination with dDG lesions without spatial contingency}

The probe experiment above showed that discriminating two objects in a fixed spatial location (i.e., arm 4) was unaffected by dDG lesions. However, there is a possibility that the intact performance was due to the reduction in the number of conditions that needed to be processed in the simple object-discrimination task compared with the biconditional task (which involved two objects $\times$ two places). To test this possibility, in Experiment 2 we created the same experimental conditions as in Experiment 1 so that rats visited two different arms (arms 3 and 5) and performed object discriminations in both arms. The only difference was that the objects used in arm 3 were never presented in arm 5 and vice versa for the objects associated with arm 5 (Fig. 1). Since all four objects were different from each other, the rats were not required to use spatial information to discriminate objects in a given arm.

As shown in Figure 3B, the control group demonstrated almost perfect performance throughout the testing period in this task after surgery. The dDG-lesion group showed $85 \%$ correct performance on the first day of postsurgical testing, which was still above our pre-surgical criterion, but was lower than the control group's performance (since the controls were almost errorless). From day 2 onward, however, the dDG-lesion group quickly recovered the normal performance level of controls and remained at that level throughout the rest of the testing period. Due to the difference in performance between the two groups on the first day of testing and because of the perfect performance in controls, a repeated-measures ANOVA showed a barely significant difference between the two groups $\left(F_{(1,6)}=6.1, P=0.049\right)$ as well as significant effects of postsurgical day $\left(F_{(5,30)}=4.9, P<\right.$ $0.01)$ and the interaction between the lesion group and postsurgical day $\left(F_{(5,30)}=5.6, P<0.001\right)$. When the ANOVA was re-run with the first day's data excluded, no significance differences were found in the group $\left(F_{(1,6)}=0.1, P>0.5\right)$, postsurgical day $\left(F_{(4,24)}=1.5, P>0.1\right)$, and the interaction between the two $\left(F_{(4,24)}=0.6, P>0.7\right)$.

\section{Recovery of impairment with dDG lesions when spatial overlap was reduced}

We interpret the results of Experiment 1 as the evidence for the involvement of DG in disambiguating object-place paired associates. Specifically, in addition to the same pair of objects used in the task, the representations of object-place paired-associative 


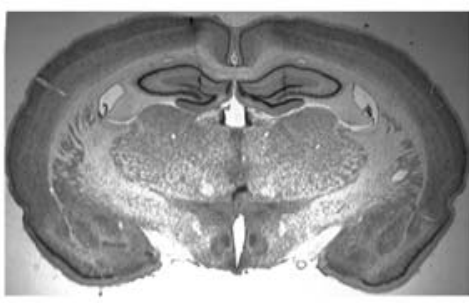

$A P-2.8 m m$

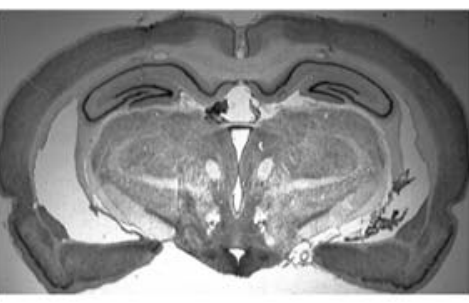

$A P-3.8 m m$
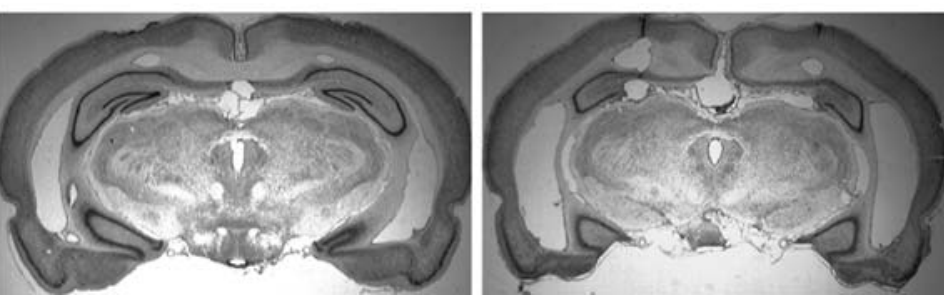

Figure 2. DG lesions. Representative photomicrographs of Nissl-stained brain sections from the control group and the DG-lesion group, sampled from three different levels along the septo-temporal dimension (AP indicates the anterior-posterior position of bregma).

events involve significant overlap between spatial contexts associated with different arms in our task. To confirm the contributions of spatial contextual overlap to performance deficits observed in dDG-lesioned animals, in Experiment 3 we maintained all of the experimental conditions, the same with Experiment 1, except that the arms were farther apart from each other (102.8 $8^{\circ}$ between arms 2 and 6$)$. The angular distance between the arms in Experiment 3 was twice as big as the distance between the arms used in Experiment 1 (51.4 $4^{\circ}$ between arms 3 and 5) to reduce the amount of overlap in spatial contexts associated with spatial locations. As in Experiment 1, the control rats were normal in performing the task after surgery (Fig. 3C). The DG-lesion group's performance was initially impaired as in Experiment 1, showing the chance level performance. However, in contrast to the sustained impairment in Experiment 1, the dDG-lesioned animals gradually relearned the task during the postsurgical training period afterward. The DG-lesion group reached $\sim 75 \%$ correct level in $6 \mathrm{~d}$, which was our pre-surgical criterion. An ANOVA with repeated measures showed highly significant effects of the lesion group $\left(F_{(1,10)}=122.9, P<0.0001\right)$ and postsurgical day $\left(F_{(5,50)}=9.3, \stackrel{P}{<}<0.0001\right)$. No interaction between the two factors was observed $\left(F_{(5,50)}=2.1, P=0.08\right)$ since performances of both groups gradually improved over time.

Comparing Figure 3, A and $\mathrm{C}$ with respect to the performances of the control groups and DG-lesion groups from both Experiments 1 and 3, there was no significant interaction between the experiment and postsurgical day between the two control groups $\left(F_{(5,50)}=1.1, P>0.1\right)$. However, the DG-lesion groups in Experiments 1 and 3 showed different learning curves after surgery (significant interaction between lesion group and postsurgical day, $\left.F_{(5,50)}=3.9, P<0.01\right)$; specifically, the dDG-lesioned rats improved performance only when the arms used in the object-place paired-associate task were far apart (Experiment 3), but not when the arms were positioned close to each other (Experiment 1). Post-hoc comparisons between the two DG-lesion groups from Experiments 1 and 3 revealed significant differences between the two groups in the later phase of the postsurgical testing period, i.e., day $4\left(t_{(10)}=-2.4\right.$, $P<0.05)$ and day $6\left(t_{(10)}=-4.2, P<\right.$ $0.01)$. The simple object-discrimination task used as a probe test at the end of Experiment 1 was also carried out in Experiment 3 for all animals after the main task, and all rats learned the task to criterion in $2 \mathrm{~d}$ (data not shown). These results suggest that, in contrast to Experiment 1, rats without dDG can learn to dissociate similar object-place paired associates if the same pair of objects appears in physically wellseparated locations in the environment.

\section{Discussion}

The involvement of the DG subfield of the hippocampus in disambiguating similar object-place paired associates has never been tested. We have demonstrated in the current study that the DG is important for processing object-place paired associates, but that its role in this cognitive domain is selective and conditional. Specifically, the DG was necessary in discriminating multiple, similar object-place paired associates when locations associated with the objects were relatively close to each other as in Experiment 1. If the ambiguity disappeared either because objects appearing in those locations were distinctively different from each other (Experiment 2) or because spatial locations were farther apart (Experiment 3), the DG-lesioned rats performed normally (Experiment 2) or relearned the task (Experiment 3).

One of the important dimensions of episodic memory is to process objects in association with their locations (Smith and Milner 1989; Abrahams et al. 1997; Vargha-Khadem et al. 1997; Nunn et al. 1998). This component of episodic memory has been studied also in animal models (Gaffan 1994; Gilbert and Kesner 2002; Rolls et al. 2005; Bachevalier and Nemanic 2008; Lee and Solivan 2008). A common focus of prior studies has been to test whether distinct objects and places can be associated as part of an event. However, less attention has been paid to investigating the mechanisms for encoding and retrieving representations composed of similar, yet different object-place paired associates. It is conceivable that numerous representations for different events or episodes can be generated within a limited spatial environment by rearranging the positions of objects in the environment. The intrahippocampal mechanisms contributing to such cognitive processes are poorly understood. Although the role of DG in pattern separation has been mostly studied in spatial domain experimentally (Gilbert et al. 2001; Leutgeb et al. 2007), it does not need to be limited to spatial domain theoretically (O'Reilly and McClelland 1994) and may be applied to hippocampal event representation (e.g., object-place association) in general.

In light of the role of DG in spatial pattern separation suggested by computational models, the results of the current study 


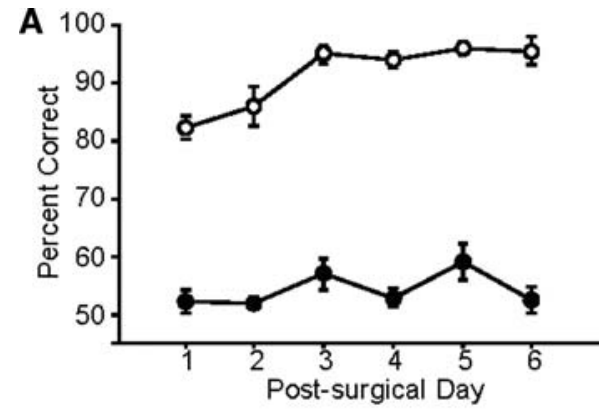

B
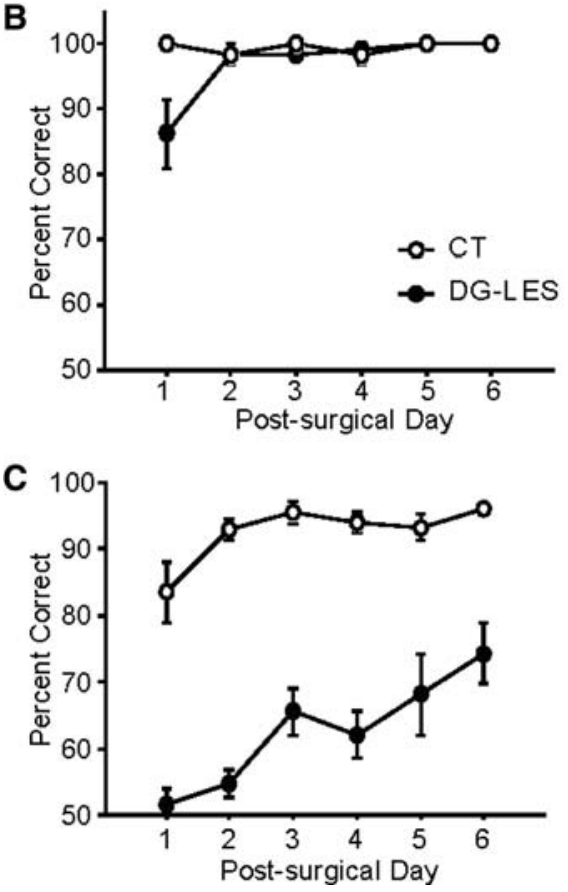

Figure 3. Postsurgical performance. (A) Performance of control $(O)$ and DG-lesioned rats $(\bullet)$ across $6 \mathrm{~d}$ of postsurgical testing in Experiment 1. (B) Postsurgical performance in Experiment 2. (C) Postsurgical performance in Experiment 3.

may be explained as shown in Figure 4. As both object and arm/ spatial information are fed to the hippocampus from extrahippocampal cortices, DG augments differences in those input patterns, especially when there is a significant overlap (e.g., overlapping contextual cues associated with arms 3 and 5 in Experiment 1), but less so when the inputs are relatively well-separated as in Experiment 3. It is unclear whether the DG also performs this pattern separation function for purely nonspatial input patterns such as objects and odors. On the basis of the results from Experiments 1 and 2 in our study, we speculate that the DG might be involved in pattern separation only when those nonspatial items appear at multiple locations and when they need to be associated with different spatial locations to form discrete object-place pairedassociative representations. However, this type of purely spatial and contextual explanation (Fig. 4) assumes that object information is unaltered as a result of the DG lesions, which may not be true, as the dDG-lesioned rats were still relatively impaired compared with controls in Experiment 3 in our study. Therefore, further testing with only nonspatial items is necessary to confirm that the DG exists mainly for spatial pattern separation.

For spatial pattern separation, Gilbert et al. (2001) showed that rats with lesions in DG were normal in discriminating two locations that were separated 82.5 and $105 \mathrm{~cm}$ apart from each other, whereas they were impaired in discriminating two locations when the distance between them was in the range of from 15 to $60 \mathrm{~cm}$. It is a possibility that not being able to discriminate arm 3 from arm 5 (spatially) contributed to the performance deficits in our task in dDG-lesioned rats (Fig. 4). However, the following observations and results from our study may warrant some cautions for the purely spatial interpretations. First, the two choice platforms of arms 3 and 5 in our task were separated by $80 \mathrm{~cm}$ and those for arms 2 and 6 were $140 \mathrm{~cm}$ apart. Although it is difficult to compare directly the distances measured in an open field vs. radial-arm maze, the choice platforms associated with arms 3 and 5 used in the task were reasonably separated. Second, although dDG-lesioned rats learned the task when the two arms (arms 2 and 6) were well separated (compared with Experiment 1 using arms 3 and 5), the animals never improved their performance to the level of controls. Considering the results of the Gilbert et al. (2001) study, this pattern of deficits suggests that lesions in dDG affected cognitive processes beyond mere spatial pattern separation between arms. It appears that the rats with lesions in dDG still had difficulty in associating the same objects with different locations and disambiguating those object-place paired associates later, even when there was minimal spatial overlap between the two arms with which objects were associated. To reach a stronger conclusion, however, it may be necessary to test the animals in a purely spatial paradigm in the same maze.

To our knowledge, the only study that has tested the role of DG in an object-place paired-associative condition is the Gilbert and Kesner (2003) study. In that study, rats were required to visit two different locations and one of two different objects was presented in each location per trial. An object was only rewarded in a particular location but not in the other location. CA3 lesions produced impairment in performance in the Gilbert and Kesner study (2003), which suggests that the results from the current study might have also been mediated by the CA3 not receiving normal inputs from the DG. In the Gilbert and Kesner study, however, it is surprising that the DG-lesioned rats were normal in learning the task as compared with control rats, and only CA3-lesioned rats were impaired. Moreover, CA3-lesioned animals did relearn the task to criterion in that study. These results suggest that the computational load for DG and CA3 for event disambiguation might have been minimal in the Gilbert and Kesner study because the rat always encountered only a single object in a given location per trial. Specifically, the rat never saw that the two objects appeared simultaneously, because the testing paradigm was a go/no-go task. In addition, the two spatial locations were fairly far apart $\left(\sim 80^{\circ}-90^{\circ}\right.$ when calculated based on the dimensions of the maze provided in the Gilbert and Kesner study) as in our Experiment 3. The rats in that study, therefore, might have formed representations for the four discrete object-place representations (associated with go/no-go responses) and could have minimal interference among those representations. By comparison, both objects in our study were simultaneously presented in different locations (51.4 ${ }^{\circ}$ apart from each other) in Experiment 1 , and the task produced severe performance deficits in DG-lesioned animals. In our Experiment 3, when the same pair of objects was presented in well-separated locations as in the Gilbert and Kesner study (2003), the DG-lesioned rats showed initial performance deficit but significantly improved their performance across days to normal level (compared with Experiment 1). This strongly suggests that the DG-CA3 network mediates the disambiguation of the same pair of objects simultaneously encountered at different locations. Experiment 2 clearly suggests that the ambiguity stemming from the same objects being associated with different spatial contexts plays an important role in recruiting DG (and possibly CA3), because the DG-lesioned rats were 


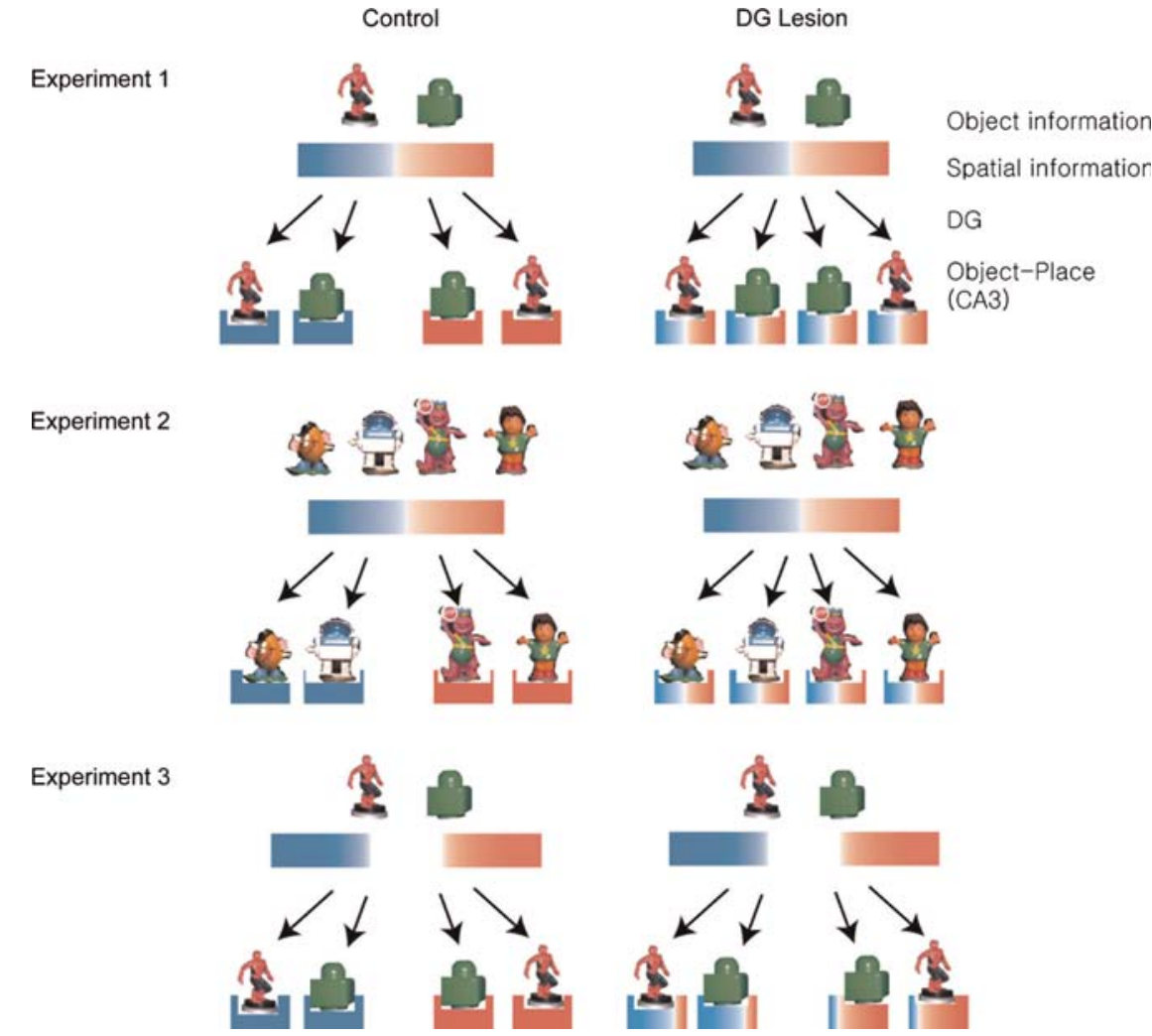

Figure 4. Hypothesized functions of DG in disambiguating object-place paired associates. The blue area in a rectangle indicates unique contextual information associated with arm 3 and the red area denotes arm 5-unique contextual information. Light gradients of blue and red areas indicate spatial contextual cues that are associated with arms 3 and 5, respectively, in an overlapping fashion. Solid blue or red rectangles represent orthogonalized representations of particular arms with no ambiguity. In Experiment 1, for example, intact DG enabled the hippocampus (CA3) to represent arms 3 and 5 into distinct spatial representations (rectangles with solid colors), whereas DG lesions prevented this and resulted in ill-separated representations for arms 3 and 5 (denoted by gradient colors). Associating the same objects with these similar spatial representations led to inseparable object-place paired associative representations and caused performance deficits in DG-lesioned animals. The illseparated spatial representations, however, did not produce performance deficits in Experiment 2, since objects were distinctively different from each other. In Experiment 3, since spatial representations of arms 2 and 6 had less overlap in contextual cues (indicated by more areas with solid colors with less gradients), the absence of DG initially produced deficits, but the remaining hippocampal circuits might have learned to use the relatively well-segregated spatial representations.

unimpaired in performing the task when there was no overlap in object identities between arms 3 and 5 .

The following alternative interpretations of the data can be also considered for future studies. First, it has been shown that object-place paired associations are disrupted by dorsal CA3 lesions (Gilbert and Kesner 2003; Kesner et al. 2008). Since DG has a direct feed-forward connection with CA3, it is possible that DG modulates the paired associative function of CA3 via a pattern separation mechanism. Second, DG may have modulated the function of CA1 for a temporal order memory for spatial location, since it has been shown that spatial pattern separation can influence or modulate temporal order memory (Hunsaker et al. 2008). These possibilities may not require DG as the main substrate for the operation of object-place associations and eliminate that one proves that object-place associations are mediated by DG.

The firing correlates of granule cells in DG have been mostly studied in spatial or contextual behavioral paradigms (Jung and McNaughton 1993; Leutgeb et al. 2007). However, only very few studies, if any, examined the firing correlates of granule cells in DG in mnemonic tasks that involved nonspatial items in conjunction with spatial locations. Our study provides compelling evidence that the DG is involved in forming and retrieving paired associates between objects and their locations in a selective manner. Future physiological studies and computational modeling of DG functions (in connection with other hippocampal subfields as well as extrahippocampal regions) using the current paradigm should contribute to elucidating the neural mechanisms underlying the formation and retrieval of event memory. Furthermore, it would be interesting in the future to test whether DG is involved in the acquisition of the similar objectplace paired associates (as in the retrieval in the current task) by producing lesions first and then training the animals in the task.

\section{Materials and Methods}

\section{Subjects}

Thirty-two male Long-Evans rats $(320-420 \mathrm{~g})$ were used in the study (Experiment 1: $n=12$, Experiment 2: $n$ $=8$, Experiment 3: $n=12$ ), each housed in a standard rodent cage. Each rat was only used for one of the experiments. The rats were maintained on a 12 -h light/dark cycle. Each rat's weight was maintained at $80 \%$ of its free-feeding weight. Water was provided ad libitum. All experimental and surgical protocols conformed to the NIH Guide for the Care and Use of Laboratory Animals and the Institutional Animal Care and Use Committee of the University of Iowa.

\section{Behavioral apparatus}

Detailed descriptions of the maze can be found in our previous work (Lee and Solivan 2008) and will only be described briefly here. A 7-arm radial maze (angular distance between adjacent arms $=25.7^{\circ}$ ) was used (Fig. 1). Seven identical arms (arms 1-7 clockwise in Fig. 1) radiated from a circular center stage in which a rectangular start box was located. At the end of each arm, a rectangular choice platform was available and three food wells were available in each choice platform. Each food well could contain a piece of cereal reward and objects were placed over the left and right food wells during behavioral testing (the middle food well was never used throughout the experiments). The maze was placed in a circular curtained area and multiple hanging cues were available along the curtains as distal visual cues. Throughout the main experiments, only two arms of the maze (arms 3 and 5 in Experiments 1 and 2, and arms 2 and 6 in Experiment 3) were used.

\section{Behavioral tasks}

Detailed pretraining procedures for handling, familiarization, and shaping for rats can be found in our previous publication (Lee and Solivan 2008). After the rats learned how to displace an object overlying the food well through the pretraining stage, training for one of the following behavioral tasks ensued (Fig. 1). 


\section{Experiment 1}

Either arm 3 or arm 5 was opened to the rat in a given trial (32 trials/day). Two toy objects (Spider-Man and LEGO block) were prearranged (food well locations associated with the two objects were counterbalanced between trials in a given arm) in the choice platform of the open arm. There were three food wells in each event platform and the two objects were positioned over the left and right food wells (left and right positions were counterbalanced between trials). One of the food wells occupied by the toy objects contained a cereal reward. The rats were required to learn a correct object rewarded in each arm to obtain reward: In arm 3, only Spider-Man was rewarded; and the LEGO block, but not Spider-Man, was rewarded in arm 5. Once a correct object was displaced, the rats were allowed to grab the cereal reward in the food well and went back to the start box to consume the reward. In contrast, when a wrong object was displaced instead, the animals were not allowed to displace the other correct object and returned to the start box with no reward. The rats were trained until they reached $\geq 75 \%$ correct performance for two consecutive days.

\section{Experiment 2}

The same procedures were used as in Experiment 1, except that four different objects were presented (two of them associated with arm 3, with the other two associated with arm 5). Specifically, a toy cartoon figure (Mr. Potato Head) and a robot toy object were used (Mr. Potato Head was always rewarded) in arm 3 and Barney and a toy doll were used in arm 5 (Barney was always rewarded).

\section{Experiment 3}

All procedures were identical with Experiment 1, except that arms 2 and 6 were used instead of arms 3 and 5 .

\section{Surgery}

Once the rat was trained to criterion, the animal was randomly assigned to either control lesion group or neurotoxic DG-lesion group and was subject to the surgical procedures previously described elsewhere in detail (Lee and Kesner 2004a,b; Lee and Solivan 2008). Briefly, after an incision was made in the scalp, five small burr holes were drilled in each hemisphere in the skull and either phosphate-buffered saline (PBS) or colchicine (7 mg/mL; Sigma-Aldrich) was injected into the dorsal hippocampus $(0.07 \mu \mathrm{L}$ at $10 \mu \mathrm{L} / \mathrm{h}$ per hole) using the following coordinates: (1) bregma $-2.3 \mathrm{~mm}$, midline $\pm 1.0 \mathrm{~mm}$, skull surface $-4.4 \mathrm{~mm}$, (2) bregma $-3.0 \mathrm{~mm}$, midline $\pm 1.4 \mathrm{~mm}$, skull surface $-4.4 \mathrm{~mm}$, (3) bregma $-4.0 \mathrm{~mm}$, midline $\pm 2.0 \mathrm{~mm}$, skull surface $-4.2 \mathrm{~mm}$, (4) bregma $-4.8 \mathrm{~mm}$, midline $\pm 3.1 \mathrm{~mm}$, skull surface $-4.3 \mathrm{~mm}$, and (5) bregma $-5.7 \mathrm{~mm}$, midline $\pm 3.8 \mathrm{~mm}$, skull surface $-4.4 \mathrm{~mm}$. Injections were made in the dorsal hippocampus.

Postsurgical testing and a simple object-discrimination task After a week of recovery, the rats were retested for $6 \mathrm{~d}$, consecutively, in the same task used for training before surgery. In Experiments 1 and 3, after the rats were tested for $6 \mathrm{~d}$, they were trained in a simple object-discrimination task, in which two toy objects (oil can and a toy doll) were presented in arm 4 only (32 trials) and the toy doll was always rewarded. The rats were trained until they reached $\geq 75 \%$ correct performance for two consecutive days.

\section{Histology}

Histological verifications of lesions and estimation of volumetric damage were performed as previously described in detail elsewhere (Gilbert et al. 2001; Lee and Kesner 2004a,b; Lee and Solivan 2008). Briefly, rats received a lethal dose of sodium pentobarbital (Euthasol, Henry Shein), followed by a transcardial infusion of $0.9 \%$ saline and a $10 \%$ formaldehyde solution. Each brain was stored in a $10 \%$ formalin-30\% sucrose solution at $4^{\circ} \mathrm{C}$ for $72 \mathrm{~h}$. The brains were frozen, cut in coronal sections $(40 \mu \mathrm{m})$ on a sliding microtome (Microm, Thermo Fisher Scientific), and later stained with thionin (Sigma).

The amount of damage in the hippocampus was threedimensionally visualized and quantified for each rat, using computer software as previously reported (Lee and Kesner 2003, 2004a,b; Lee and Solivan 2008). For this procedure, the brain sections were digitally captured via Photoshop (Adobe systems, Inc.) and the boundaries of the major cell layers in the hippocampus (i.e., pyramidal cell layers in CA regions and granule cell layers in the dentate gyrus) were drawn using a pen tablet as an input device (Wacom). This was performed in tandem with the examination of the original slides under the microscope at high magnification $(\geq X 40)$ for accurate delineations of the intact cell boundaries. The two-dimensional images of the cell layers delineated were detached as separate image layers from the original, digital section images in Photoshop and converted to black-and-white bitmap images. The serial sections of bitmap images were then three-dimensionally reconstructed (Voxwin). Comparing the number of voxels used for reconstructing the major cell layers in the dorsal hippocampus in the DG-lesion and control groups provided a volumetric estimation of the damage produced in the dorsal DG by colchicine.

\section{Acknowledgments}

The current study was supported by the WCU program of the Ministry of Education, Science, and Technology in Korea through KOSEF (R32-10142) and by NIMH RO1 MH079971. We thank Amy Handley, Sarah Knight, Jacque Tribbett, Melissa Marchal, and Janice Kim for assisting in behavioral data collection and Jayoung Byun for helping with the data analysis.

\section{References}

Abrahams S, Pickering A, Polkey CE, Morris RG. 1997. Spatial memory deficits in patients with unilateral damage to the right hippocampal formation. Neuropsychologia 35: 11-24.

Bachevalier J, Nemanic S. 2008. Memory for spatial location and object-place associations are differently processed by the hippocampal formation, parahippocampal areas $\mathrm{TH} / \mathrm{TF}$ and perirhinal cortex. Hippocampus 18: 64-80.

Gaffan D. 1994. Scene-specific memory for objects: A model of episodic memory impairment in monkeys with fornix transection. J Cogn Neurosci 6: 305-320.

Gaffan D, Parker A. 1996. Interaction of perirhinal cortex with the fornix-fimbria: Memory for objects and "object-in-place" memory. J Neurosci 16: 5864-5869.

Gilbert PE, Kesner RP. 2002. Role of the rodent hippocampus in paired-associate learning involving associations between a stimulus and a spatial location. Behav Neurosci 116: 63-71.

Gilbert PE, Kesner RP. 2003. Localization of function within the dorsal hippocampus: The role of the CA3 subregion in paired-associate learning. Behav Neurosci 117: 1385-1394.

Gilbert PE, Kesner RP, Lee I. 2001. Dissociating hippocampal subregions: Double dissociation between dentate gyrus and CA1. Hippocampus 11: 626-636.

Hunsaker MR, Fieldsted PM, Rosenberg JS, Kesner RP. 2008. Dissociating the roles of dorsal and ventral CA1 for the temporal processing of spatial locations, visual objects, and odors. Behav Neurosci 122: 643-650.

Jerman TS, Kesner RP, Lee I, Berman RF. 2005. Patterns of hippocampal cell loss based on subregional lesions of the hippocampus. Brain Res 1065: $1-7$.

Jung MW, McNaughton BL. 1993. Spatial selectivity of unit activity in the hippocampal granular layer. Hippocampus 3: 165-182.

Kesner RP, Hunsaker MR, Warthen MW. 2008. The CA3 subregion of the hippocampus is critical for episodic memory processing by means of relational encoding in rats. Behav Neurosci 122: 1217-1225.

Kim JJ, Fanselow MS. 1992. Modality-specific retrograde amnesia of fear. Science 256: 675-677.

Lee I, Kesner RP. 2003. Differential roles of dorsal hippocampal subregions in spatial working memory with short vs. intermediate delay. Behav Neurosci 117: 1044-1053.

Lee I, Kesner RP. 2004a. Differential contributions of dorsal hippocampal subregions to memory acquisition and retrieval in contextual fear-conditioning. Hippocampus 14: 301-310. 
Lee I, Kesner RP. 2004b. Encoding vs. retrieval of spatial memory: Double dissociation between the dentate gyrus and the perforant path inputs into CA3 in the dorsal hippocampus. Hippocampus 14: $66-76$.

Lee I, Solivan F. 2008. The roles of the medial prefrontal cortex and hippocampus in a spatial paired-association task. Learn Mem 15: 357-367.

Leutgeb JK, Leutgeb S, Moser M-B, Moser EI. 2007. Pattern separation in the dentate gyrus and CA3 of the Hippocampus. Science 315: 961-966.

Marr D. 1971. Simple memory: A theory for archicortex. Philos Trans R Soc Lond B Biol Sci 262: 23-81.

McHugh TJ, Jones MW, Quinn JJ, Balthasar N, Coppari R, Elmquist JK, Lowell BB, Fanselow MS, Wilson MA, Tonegawa S. 2007. Dentate gyrus NMDA receptors mediate rapid pattern separation in the hippocampal network. Science 317: 94-99.

McNaughton BL, Morris RGM. 1987. Hippocampal synaptic enhancement and information storage within a distributed memory system. Trends Neurosci 10: $408-415$.

Mundy WR, Tilson HA. 1990. Neurotoxic effects of colchicine. Neurotoxicology 11: 539-547.

Nunn JA, Polkey CE, Morris RG. 1998. Selective spatial memory impairment after right unilateral temporal lobectomy. Neuropsychologia 36: $837-848$.
O'Keefe J, Nadel L. 1978. The hippocampus as a cognitive map. Clarendon Press, Oxford, UK.

O'Reilly RC, McClelland JL. 1994. Hippocampal conjunctive encoding, storage, and recall: Avoiding a trade-off. Hippocampus 4: 661-682.

Rolls ET, Xiang J, Franco L. 2005. Object, space, and object-space representations in the primate hippocampus. J Neurophysiol 94: 833-844.

Smith ML, Milner B. 1989. Right hippocampal impairment in the recall of spatial location: Encoding deficit or rapid forgetting? Neuropsychologia 27: 71-81.

Treves A, Rolls ET. 1992. Computational constraints suggest the need for two distinct input systems to the hippocampal CA3 network. Hippocampus 2: 189-199.

Vargha-Khadem F, Gadian DG, Watkins KE, Connelly A, Van Paesschen W, Mishkin M. 1997. Differential effects of early hippocampal pathology on episodic and semantic memory. Science 277: 376-380.

Xavier GF, Oliveira-Filho FJ, Santos AM. 1999. Dentate gyrus-selective colchicine lesion and disruption of performance in spatial tasks: Difficulties in "place strategy" because of a lack of flexibility in the use of environmental cues? Hippocampus 9: 668-681.

Received October 21, 2009; accepted in revised form February 19, 2010. 


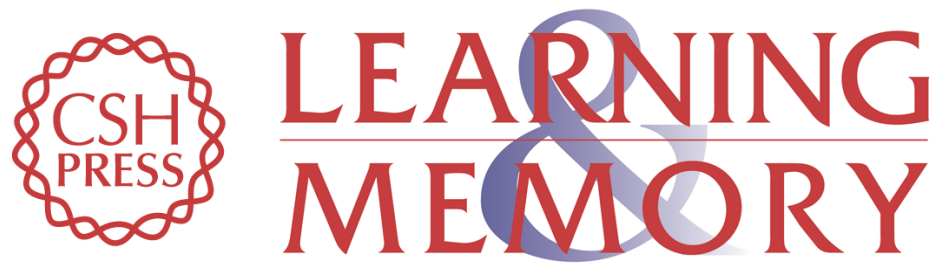

\section{Dentate gyrus is necessary for disambiguating similar object-place representations}

Inah Lee and Frances Solivan

Learn. Mem. 2010, 17:

Access the most recent version at doi:10.1101//m.1678210

References This article cites 28 articles, 6 of which can be accessed free at:

http://learnmem.cshlp.org/content/17/5/252.full.html\#ref-list-1

License

Email Alerting Receive free email alerts when new articles cite this article - sign up in the box at the Service top right corner of the article or click here. 